PROCEEDINGS OF THE

AMERICAN MATHEMATICAL SOCIETY

Volume 135, Number 5, May 2007, Pages 1465-1475

S 0002-9939(06)08626-6

Article electronically published on November 29, 2006

\title{
POWERS AND ROOTS OF TOEPLITZ OPERATORS
}

\author{
ISSAM LOUHICHI
}

(Communicated by Joseph A. Ball)

\begin{abstract}
We study the commutativity of two Toeplitz operators whose symbols are quasihomogeneous functions. We give a relationship between this commutativity and the roots (or powers) of the Toeplitz operators. We use this to characterize Toeplitz operators with symbols in $L^{\infty}(\mathbb{D})$ which commute with Toeplitz operators whose symbols are of the form $e^{i p \theta} r^{m}$.
\end{abstract}

\section{INTRODUCTION}

Let $\mathbb{D}$ denote the open unit disk in the complex plane $\mathbb{C}$, and let $d A$ denote normalized Lebesgue area measure. The Bergman space, denoted by $L_{a}^{2}$, is the Hilbert space of analytic functions on $\mathbb{D}$ that are square integrable with respect to $d A$. It is well known that $L_{a}^{2}$ is a closed subspace of the Hilbert space $L^{2}(\mathbb{D}, d A)$ and $\left(\sqrt{n+1} z^{n}\right)_{n \in \mathbb{N}}$ is an orthonormal basis of $L_{a}^{2}$. Let $P$ be the orthogonal projection of $L^{2}(\mathbb{D}, d A)$ onto $L_{a}^{2}$. For a function $\phi \in L^{\infty}(\mathbb{D}, d A)$, the Toeplitz operator with symbol $\phi$ is the operator $T_{\phi}$ from $L_{a}^{2}$ to $L_{a}^{2}$ defined by $T_{\phi}(f)=P(\phi f)$.

If $k_{z}(w)=\frac{1}{(1-\bar{z} w)^{2}}=\sum_{j=0}^{\infty}(1+j) w^{j} \bar{z}^{j}$ is the Bergman reproducing kernel, then

$$
T_{\phi}(f)(z)=P(\phi f)(z)=\int_{\mathbb{D}} \phi(w) f(w) \overline{k_{z}(w)} d A(w) .
$$

The question to be studied in this paper is: When do two Toeplitz operators $T_{\phi}$ and $T_{\psi}$ commute? In 1964, Brown and Halmos [4] solved this problem for the analogously defined Toeplitz operators on the Hardy space. They showed that $T_{\phi} T_{\psi}=T_{\psi} T_{\phi}$ for some $\phi$ and $\psi \in L^{\infty}(\mathbb{T})$, where $\mathbb{T}$ is the unit circle of $\mathbb{C}$, if and only if either

(a) $\phi$ and $\psi$ are both analytic, or

(b) $\bar{\phi}$ and $\bar{\psi}$ are both analytic, or

(c) one of the two symbols is a linear function of the other.

We recall that a function in $L^{\infty}(\mathbb{T})$ is said to be analytic if all of its Fourier coefficients with negative indices are equal to 0 .

The same question concerning Toeplitz operators on the Bergman space has a much more complicated answer. There are however some results which resemble those of [4. In fact, Axler and Cucucković proved in 2] that the condition that one of (a), (b) or (c) be true is still necessary and sufficient when the two symbols $\phi$ and $\psi$ are bounded harmonic functions on $\mathbb{D}$. Moreover, with Rao [3], they proved

Received by the editors March 26, 2005 and, in revised form, December 20, 2005.

2000 Mathematics Subject Classification. Primary 47B35; Secondary 47L80.

Key words and phrases. Toeplitz operators, Bergman space, Mellin transform.

(C)2006 American Mathematical Society Reverts to public domain 28 years from publication 1465 
that if $\phi$ is a bounded analytic function and if $\psi$ is a bounded symbol such that $T_{\phi}$ and $T_{\psi}$ commute, then $\psi$ must be analytic too. When we consider arbitrary symbols, things are different. In [5] Cučković and Rao used the Mellin transform to study the commutativity of multiplication of two Toeplitz operators $T_{\phi}$ and $T_{\psi}$ on the Bergman space and describe those operators which commute with $T_{e^{i p \theta} r^{m}}$ for $(m, p) \in \mathbb{N} \times \mathbb{N}$. In this paper we use our results from [7] to interpret and extend the results of [5]. We give some solutions in the case where the Toeplitz operators have symbols which are "quasihomogeneous" functions and show that these solutions are related to " $p^{t h}$ roots" and powers of the Toeplitz operators.

As in [7] we say that a bounded symbol $f$ is quasihomogeneous of degree $k$ if it is of the form $e^{i k \theta} \phi$ where $\phi$ is a radial function. In this case we say that the Toeplitz operator $T_{f}$ is quasihomogeneous of degree $k$.

\section{Preliminaries}

The Mellin transform of a function $\psi \in L^{1}([0,1], r d r)$ is defined by

$$
\widehat{\psi}(z)=\int_{0}^{1} \psi(r) r^{z-1} d r .
$$

It is easy to see that $\widehat{\psi}$ is a bounded holomorphic function on the half-plane $\Pi=$ $\{z: \Re z>2\}$.

We denote the Mellin convolution of two functions $\phi$ and $\psi$ by $\phi *_{M} \psi$ and we define it by the equation

$$
\left(\phi *_{M} \psi\right)(r)=\int_{r}^{1} \phi\left(\frac{r}{t}\right) \psi(t) \frac{d t}{t} .
$$

It is clear that the Mellin transform converts Mellin convolution into a pointwise product, i.e., that

$$
\left(\widehat{\phi *_{M} \psi}\right)(r)=\widehat{\phi}(r) \widehat{\psi}(r) .
$$

We shall often use the following classical theorem (see [8, p. 102]).

Theorem 1. Suppose that $f$ is a bounded, holomorphic function on $\{z: \Re z>0\}$ which vanishes at the pairwise distinct points $d_{1}, d_{2}, \cdots$, where

i) $\inf \left\{\left|d_{n}\right|\right\}>0$ and

ii) $\sum_{n \geq 1} \Re\left(\frac{1}{d_{n}}\right)=\infty$.

Then $f$ vanishes identically on $\{z: \Re z>0\}$.

Remark 2. We shall often apply this theorem to show that if $\psi \in L^{1}([0,1], r d r)$ and if there exist $n_{0} \in \mathbb{Z}_{+}, p \in \mathbb{N}$ such that

$$
\widehat{\psi}\left(n_{0}+p k\right)=0 \text { for all } k \in \mathbb{N},
$$

then $\widehat{\psi}(z)=0$ for all $z \in\{z: \Re z>2\}$ and so $\psi=0$.

\section{Powers of Toeplitz operators}

The following lemma determines the values of powers of a bounded quasihomogeneous Toeplitz operator evaluated at any element of the orthonormal basis of $L_{a}^{2}$. 
Lemma 3. Let $n \in \mathbb{N}, s \in \mathbb{Z}_{+}$and let $\psi$ be a bounded radial function on $\mathbb{D}$. Then, for all $k \in \mathbb{N}$ we have

$$
\begin{aligned}
\left(T_{e^{i s \theta} \psi}\right)^{n}\left(\xi^{k}\right)(z) & =\left[\prod_{j=0}^{n-1} 2(k+j s+s+1) \widehat{\psi}(2 k+2 j s+s+2)\right] z^{k+n s} \\
& =\frac{\prod_{j=0}^{n-1} \widehat{\psi}(2 k+2 j s+s+2)}{\prod_{j=0}^{n-1} \widehat{\mathbb{1}}(2 k+2 j s+2 s+2)} z^{k+n s},
\end{aligned}
$$

where $\mathbb{1}$ denotes the constant function with value one.

Proof. The lemma is a consequence of the following direct calculation. We write

$$
T_{e^{i s \theta} \psi}\left(\xi^{k}\right)(z)=\int_{0}^{1} \int_{0}^{2 \pi} \psi(r) r^{k} \sum_{j=0}^{\infty}(j+1) e^{i(k+s-j) \theta} r^{j} z^{j} \frac{1}{\pi} r d r d \theta
$$

and interchange the integral over $[0,2 \pi]$ and the sum to see that

$$
\begin{aligned}
T_{e^{i s \theta} \psi}\left(\xi^{k}\right)(z) & =2(k+s+1) \widehat{\psi}(2 k+s+2) z^{k+s} \\
& =\frac{\widehat{\psi}(2 k+s+2)}{\widehat{\mathbb{1}}(2 k+2 s+2)} z^{k+s} .
\end{aligned}
$$

The lemma is proved by applying $T_{e^{i s \theta} \psi}$ to $\xi^{k} n$ times.

We have the following decomposition of $L^{2}(\mathbb{D}, d A)$ as

$$
L^{2}(\mathbb{D}, d A)=\bigoplus_{k \in \mathbb{Z}} e^{i k \theta} \mathcal{R},
$$

where $\mathcal{R}$ is the space of functions on $[0,1]$ that are square integrable with respect to the measure $r d r$. Thus every function $f \in L^{2}(\mathbb{D}, d A)$ has the decomposition

$$
f\left(r e^{i k \theta}\right)=\sum_{k=-\infty}^{+\infty} e^{i k \theta} f_{k}(r), \quad f_{k} \in \mathcal{R} .
$$

Moreover, if $f \in L^{\infty}(\mathbb{D}, d A) \subset L^{2}(\mathbb{D}, d A)$, then for each $r \in[0,1)$,

$$
\left|f_{k}(r)\right|=\frac{1}{2 \pi}\left|\int_{0}^{2 \pi} f\left(r e^{i \theta}\right) e^{-i k \theta} d \theta\right| \leq \sup _{z \in \mathbb{D}}|f(z)|, \quad \forall k \in \mathbb{Z},
$$

and so the functions $f_{k}$ are bounded in the disk.

In 7] we proved the following results, which we will use in the proof of our main theorem.

Proposition 4. Let $\phi$ be a nonzero bounded radial function, let $p$ be a positive integer and let $f\left(r e^{i k \theta}\right)=\sum_{k=-\infty}^{+\infty} e^{i k \theta} f_{k}(r) \in L^{\infty}(\mathbb{D}, d A)$. Then

a) $T_{f}$ commutes with $T_{e^{i p \theta} \phi}$ if and only if $T_{e^{i k \theta} f_{k}}$ commutes with $T_{e^{i p \theta} \phi}$ for all $k \in \mathbb{Z}$.

b) If there exists $k \in \mathbb{Z}_{-}$and a bounded radial function $f_{k}$ such that

$$
T_{e^{i p \theta} \phi} T_{e^{i k \theta} f_{k}}=T_{e^{i k \theta} f_{k}} T_{e^{i p \theta} \phi},
$$

then $f_{k}$ must be equal to zero.

c) If there exists $k \in \mathbb{Z}_{+}$and a bounded radial function $f_{k}$ such that

$$
T_{e^{i p \theta} \phi} T_{e^{i k \theta} f_{k}}=T_{e^{i k \theta} f_{k}} T_{e^{i p \theta} \phi},
$$

then $f_{k}$ is unique up to a constant factor. In particular $f_{0}$ is a constant. 
Thus if $p>0, f\left(r e^{i k \theta}\right)=\sum_{k=-\infty}^{+\infty} e^{i k \theta} f_{k}(r)$ and $T_{f}$ commutes with $T_{e^{i p \theta} \phi}$, then each $f_{k}$ is uniquely determined up to multiplication by a constant and is equal to 0 for $k<0$.

Next we present two technical but easy results which permit us to prove Propositions 7 and 9, the principal results of this section.

Remark 5. Let $\left(a_{l}\right)_{l \in \mathbb{N}}$ and $\left(b_{l}\right)_{l \in \mathbb{N}}$ be two nonvanishing sequences and $p$ and $s$ two positive integers such that

$$
a_{l+s} b_{l}=b_{l+p} a_{l} \quad \text { for all } l \in \mathbb{N} .
$$

Then if

$$
A_{k}=\prod_{j=0}^{s-1} a_{k+j p} \quad \text { and } \quad B_{k}=\prod_{j=0}^{p-1} b_{k+j s}
$$

we have

$$
A_{k} B_{k+p}=A_{k+p} B_{k} \quad \text { for all } k \in \mathbb{N} .
$$

(Just multiply the $p$ equations obtained by taking $l=k, k+s, . ., k+(p-1) s$ in (2) together to see that, if (2) is true, then

$$
\frac{B_{k+p}}{B_{k}}=\frac{a_{k+p s}}{a_{k}}=\frac{A_{k+p}}{A_{k}} \quad \text { for all } k \in \mathbb{N} \text {.) }
$$

Notation. Let $S$ and $T$ be two functions (resp. two operators). We will say that $S \equiv T$ if there exists a constant $c \neq 0$ such that $S=c T$.

Lemma 6. Let $F$ and $G$ be two nonzero bounded holomorphic functions on the half plane $\Pi=\{z: \Re z>2\}$. If there exists $p \in \mathbb{N}$ such that

$$
F(z) G(z+p)=F(z+p) G(z) \text { for all } z \in \Pi,
$$

then $F \equiv G$.

Proof. Suppose that (3) is true. Then, if (as above) we multiply the $k$ equations obtained by taking $z_{n}=z+n p$ for $n=0, \ldots, k-1$, we have

$$
F(z) G(z+k p)=F(z+k p) G(z) \text { for all } k \in \mathbb{N} \text {. }
$$

Now, let $z_{0} \in \Pi$ such that $G\left(z_{0}\right) \neq 0$ and let $E=\left\{k \in \mathbb{N}: G\left(z_{0}+k p\right)=0\right\}$. If $\sum_{k \in E} \Re\left(\frac{1}{\left|z_{0}+k p\right|}\right)=\infty$, then Theorem 1 implies that $G=0$. This contradicts the hypothesis of the lemma. Thus $\sum_{k \in E^{c}} \Re\left(\frac{1}{\left|z_{0}+k p\right|}\right)=\infty$, where $E^{c}$ is the complement in $\mathbb{N}$ of the set $E$.

Now, equation (4) implies that

$$
\frac{F\left(z_{0}+k p\right)}{G\left(z_{0}+k p\right)}=\frac{F\left(z_{0}\right)}{G\left(z_{0}\right)} \text { for all } k \in E^{c} .
$$

So, applying Theorem 1 to the function $F-c G$, where $c=\frac{F\left(z_{0}\right)}{G\left(z_{0}\right)}$, completes the proof.

Let $p$ and $s$ be two positive integers and $\psi$ a bounded radial function.

If $\left(T_{e^{i s \theta} \psi}\right)^{p}$ is a Toeplitz operator, then it is the unique quasihomogeneous Toeplitz operator of degree ps (see Proposition 3 and Proposition 4 of [7]) which commutes with $T_{e^{i s \theta} \psi}$. It is natural to ask whether all nonzero Toeplitz operators which are of quasihomogeneous of degree a multiple of $s$ and which commute with $T_{e^{i s \theta} \psi}$ are of this form. 
Proposition 7. Let $p$ and $s$ be two positive integers and $\phi$ and $\psi$ be two nonzero bounded radial functions such that

$$
T_{e^{i p \theta} \phi} T_{e^{i s \theta} \psi}=T_{e^{i s \theta} \psi} T_{e^{i p \theta} \phi} .
$$

Then

$$
\left(T_{e^{i p \theta} \phi}\right)^{s} \equiv\left(T_{e^{i s \theta} \psi}\right)^{p}
$$

Proof. For all $k \in \mathbb{N}$, let

$$
a_{k}=\frac{\widehat{\phi}(2 k+p+2)}{\widehat{\mathbb{1}}(2 k+2 p+2)} \quad \text { and } \quad b_{k}=\frac{\widehat{\psi}(2 k+s+2)}{\widehat{\mathbb{1}}(2 k+2 s+2)}
$$

so that

$$
T_{e^{i p \theta} \phi}\left(\xi^{k}\right)(z)=a_{k} z^{k+p} \quad \text { and } \quad T_{e^{i s \theta} \psi}\left(\xi^{k}\right)(z)=b_{k} z^{k+s} \text {. }
$$

Then equation (5) shows that $a_{k+s} b_{k}=b_{k+p} a_{k}$ for all $k \in \mathbb{Z}_{+}$, and so Remark 5 implies that

$$
\prod_{j=0}^{s-1} a_{k+j p} \prod_{j=0}^{p-1} b_{k+p+j s}=\prod_{j=0}^{s-1} a_{k+p+j p} \prod_{j=0}^{p-1} b_{k+j s} .
$$

Let $F$ and $G$ be the two bounded holomorphic functions defined for all $z \in \Pi$ by

$$
F(z)=\prod_{j=0}^{p-1} \widehat{\mathbb{1}}(z+2 j s+2 s) \prod_{j=0}^{s-1} \widehat{\phi}(z+2 j p+p)
$$

and

$$
G(z)=\prod_{j=0}^{s-1} \widehat{\mathbb{1}}(z+2 j p+2 p) \prod_{j=0}^{p-1} \widehat{\psi}(z+2 j s+s) .
$$

Then equation (7) is equivalent to

$$
F(2 k+2) G(2 k+2 p+2)=F(2 k+2 p+2) G(2 k+2) \quad \text { for all } k \in \mathbb{Z}_{+} .
$$

Now, applying Theorem 1, in the form of Remark 2, implies that

$$
F(z) G(z+2 p)=F(z+2 p) G(z) \quad \text { for all } z \in \Pi \text {. }
$$

Finally, using Lemma 6, we obtain that

$$
\frac{\prod_{j=0}^{s-1} \widehat{\phi}(z+2 j p+p)}{\prod_{j=0}^{s-1} \widehat{\mathbb{1}}(z+2 j p+2 p)} \equiv \frac{\prod_{j=0}^{p-1} \widehat{\psi}(z+2 j s+s)}{\prod_{j=0}^{p-1} \widehat{\mathbb{1}}(z+2 j s+2 s)} \quad \text { for all } z \in \Pi,
$$

and Lemma 3 completes the proof.

Remark 8. i) We will assume that $\left(T_{e^{i p \theta} \phi}\right)^{0}=I$, where $I$ is the identity operator of $L_{a}^{2}$ onto $L_{a}^{2}$.

ii) If $p$ and $s$ are both negative integers and if $T_{e^{i p \theta} \phi} T_{e^{i s \theta} \psi}=T_{e^{i s \theta} \psi} T_{e^{i p \theta} \phi}$, then by considering the adjoint operators we obtain

$$
T_{e^{-i s \theta} \psi} T_{e^{-i p \theta} \phi}=T_{e^{-i p \theta} \phi} T_{e^{-i s \theta} \psi}
$$

and so Proposition 7 implies that $\left(T_{e^{-i p \theta} \phi}\right)^{-s} \equiv\left(T_{e^{-i s \theta} \psi}\right)^{-p}$.

Now, by considering once again the adjoint operators we see that

$$
\left(T_{e^{i p \theta} \phi}\right)^{-s} \equiv\left(T_{e^{i s \theta} \psi}\right)^{-p} \text {. }
$$


Proposition 9. Let $\phi$ and $\psi$ be two nonzero bounded radial functions and $n, p$ and $s$ be positive integers. Then

$$
\left(T_{e^{i p s \theta} \phi}\right)^{n}=\left(T_{e^{i s \theta} \psi}\right)^{n p} \Longrightarrow T_{e^{i p s \theta} \phi}=\left(T_{e^{i s \theta} \psi}\right)^{p} .
$$

Proof. For all $k \in \mathbb{Z}_{+}$, let

$$
a_{k}=2(k+p s+1) \widehat{\phi}(2 k+p s+2) \quad \text { and } \quad b_{k}=2(k+s+1) \widehat{\psi}(2 k+s+2),
$$

so that

$$
\left(T_{e^{i p s \theta} \phi}\right)^{n}=\left(T_{e^{i s \theta} \psi}\right)^{n p} \Leftrightarrow \prod_{j=0}^{n-1} a_{k+j p s}=\prod_{j=0}^{n p-1} b_{k+j s} \quad \text { for all } k \in \mathbb{Z}_{+}
$$

and

$$
T_{e^{i p s \theta} \phi}=\left(T_{e^{i s \theta} \psi}\right)^{p} \Leftrightarrow a_{k}=\prod_{j=0}^{p-1} b_{k+j s} \quad \text { for all } k \in \mathbb{Z}_{+} .
$$

Suppose that

$$
\prod_{j=0}^{n-1} a_{k+j p s}=\prod_{j=0}^{n p-1} b_{k+j s} \quad \text { for all } k \in \mathbb{Z}_{+} .
$$

We will prove that

$$
a_{k n p s}=\prod_{j=0}^{p-1} b_{k n p s+j s} \quad \text { for all } k \in \mathbb{Z}_{+} .
$$

We prove (9) by induction on $k$. If we take $k=0$ in equation (8), then we obtain

$$
\begin{aligned}
\prod_{j=0}^{n-1} a_{j p s} & =\prod_{j=0}^{n p-1} b_{j s} \\
& =\prod_{j=0}^{p-1} b_{j s} \prod_{j=p}^{n p-1} b_{j s} \\
& =\prod_{j=0}^{p-1} b_{j s} \prod_{j=0}^{n p-1} b_{p s+j s} .
\end{aligned}
$$

Otherwise

$$
\begin{aligned}
\prod_{j=0}^{n-1} a_{j p s} & =a_{0} \prod_{j=1}^{n-1} a_{j p s} \\
& =a_{0} \prod_{j=0}^{n-1} a_{p s+j p s} .
\end{aligned}
$$

But equation (8) implies that

$$
\prod_{j=0}^{n-1} a_{p s+j p s}=\prod_{j=0}^{n p-1} b_{p s+j s} .
$$

Thus

$$
a_{0}=\prod_{j=0}^{p-1} b_{j s}
$$


Now, assume (9) is true for knps. We show it is true for $(k+1) n p s$. We set $k$ equal to knps in the left-hand side of (8) and obtain

$$
\prod_{j=0}^{n-1} a_{k n p s+j p s}=a_{k n p s} \prod_{j=0}^{n-2} a_{k n p s+p s+j p s} .
$$

Then

$$
a_{(k+1) n p s} \prod_{j=0}^{n-1} a_{k n p s+j p s}=a_{k n p s} \prod_{j=0}^{n-1} a_{k n p s+p s+j p s} .
$$

But

$$
\prod_{j=0}^{n-1} a_{k n p s+p s+j p s}=\prod_{j=0}^{n p-1} b_{k n p s+p s+j s}
$$

and

$$
\prod_{j=0}^{n p-1} b_{k n p s+p s+j s} \prod_{j=0}^{p-1} b_{k n p s+j s}=\prod_{j=0}^{n p-1} b_{k n p s+j s} \prod_{j=0}^{p-1} b_{(k+1) n p s+j s} .
$$

Thus (9) is proved for $(k n p s)_{k \in \mathbb{Z}_{+}}$. Hence, for all $k \in \mathbb{Z}_{+}$, we have

$$
\widehat{\phi}(2 k n p s+p s+2) \prod_{j=0}^{p-1} \widehat{\mathbb{1}}(2 k n p s+2 j s+2 s+2)=\widehat{\mathbb{1}}(2 k n p+p+2) \prod_{j=0}^{p-1} \widehat{\psi}(2 k n p+1+2)
$$

and, using equation (11) and Remark 2, we complete the proof.

Remark 10. In [7] (Proposition 6) we prove that if $p>0$ and $\phi$ is a nonzero bounded radial function and if there exists a bounded radial function $\psi$ such that $T_{\psi}$ commutes with $T_{e^{i p \theta} \phi}$, then $\psi$ must be a constant. Here is another proof of this proposition. In fact, using Proposition 7 , we have $\left(T_{\psi}\right)^{p} \equiv I$, so Proposition 9 implies that $T_{\psi} \equiv I$, and so, that $\psi \equiv \mathbb{1}$ since $I$ is the Toeplitz operator of symbol $\mathbb{1}$.

\section{MAin RESUlt}

Let $p$ be a positive integer. We start this section with the definition of the T- $p^{t h}$ root of a quasihomogeneous Toeplitz operator of degree $p$ or $-p$. This new notion plays an important role in the remainder of the paper.

Definition 11. Let $\phi$ be a nonzero bounded radial function and $p$ be a positive integer. We say that the Toeplitz operator $T_{e^{i p \theta} \phi}$ has a T- $p^{t h}$ root $T_{e^{i \theta} \psi}$ if and only if there exists a nonzero bounded radial function $\psi$ such that

$$
T_{e^{i p \theta} \phi}=\left(T_{e^{i \theta} \psi}\right)^{p} \text {. }
$$

Remark 12. i) The T- $p^{\text {th }}$ root of a quasihomogeneous Toeplitz operator is unique. In fact, suppose that $T_{e^{i p \theta} \phi}$ has two T- $p^{t h} \operatorname{roots} T_{e^{i \theta} \psi}$ and $T_{e^{i \theta}} \widetilde{\psi}$. Then $\left(T_{e^{i \theta} \psi}\right)^{p}=\left(T_{e^{i \theta} \widetilde{\psi}}\right)^{p}$. Then, by Proposition 9, we have that $T_{e^{i \theta} \psi}=$ $T_{e^{i \theta} \tilde{\psi}}$, which implies that $\psi=\widetilde{\psi}$.

ii) If the quasihomogeneous degree is negative we have an analogous definition of the T- $p^{\text {th }}$ root. Let $p$ be a positive integer and $\phi$ be a bounded radial function. Then, we say that $T_{e^{-i p \theta} \phi}$ has a T- $p^{\text {th }}$ root if there exists a bounded radial function $\psi$ such that $T_{e^{-i p \theta} \phi}=\left(T_{e^{-i \theta} \psi}\right)^{p}$. It is easy to see, 
by taking adjoints, that $T_{e^{-i p \theta} \phi}$ has a T- $p^{t h} \operatorname{root} T_{e^{-i \theta} \psi}$ if and only if $T_{e^{i p \theta} \phi}$ has a T- $p^{t h}$ root $T_{e^{i \theta} \psi}$.

Examples. $\quad$ i) $T_{e^{i \theta}\left(\frac{r+r^{5}}{2}\right)}$ is the T-2 ${ }^{\text {th }}$ root of $T_{e^{2 i \theta} r^{6}}$.

ii) $T_{e^{i \theta}\left(\frac{3 r+2 r^{5}+3 r^{9}}{8}\right)}$ is the T-2 $2^{\text {th }}$ root of $T_{e^{2 i \theta} r^{10}}$.

Now, if $T_{e^{i \theta} \psi}$ is the T-p $p^{t h}$ root of $T_{i p \theta}$ and if $\left(T_{e^{i \theta} \psi}\right)^{k}$ (for $k$ in $\mathbb{N}$ ) is a Toeplitz operator, then $\left(T_{e^{i \theta} \psi}\right)^{k}$ is the unique nonzero quasihomogeneous Toeplitz operator of degree $k$ which can commute with $T_{e^{i p \theta} \phi}$. What we prove below is that if $T_{e^{i p \theta} \phi}$ has a $\mathrm{T}-p^{t h}$ root $T_{e^{i \theta} \psi}$, then the only nonzero quasihomogeneous Toeplitz operator of degree $s$ which commutes with $T_{e^{i p \theta} \phi}$ is an $s^{t h}$ power of $T_{e^{i \theta} \psi}$, extending the result (Propositions 7 and 9) of section 3 in this case.

Theorem 13. Let $\phi$ be a nonzero bounded radial function and let $p$ be a positive integer. Assume that $T_{e^{i p \theta} \phi}$ has a $T-p^{\text {th }}$ root $T_{e^{i \theta} \psi}$. Suppose that

$$
f\left(r e^{i \theta}\right)=\sum_{k=-\infty}^{+\infty} e^{i k \theta} f_{k}(r) \in L^{\infty}(\mathbb{D}, d A)
$$

is such that

$$
T_{f} T_{e^{i p \theta} \phi}=T_{e^{i p \theta} \phi} T_{f}
$$

Then

i) $f_{k}=0$ for $k<0$.

ii) If $k \geq 0$ and $\left(T_{e^{i \theta} \psi}\right)^{k}$ is a Toeplitz operator, then either $T_{e^{i k \theta} f_{k}} \equiv\left(T_{e^{i \theta} \psi}\right)^{k}$ or $f_{k}=0$.

iii) If $k \geq 0$ and $\left(T_{e^{i \theta} \psi}\right)^{k}$ is not a Toeplitz operator, then $f_{k}=0$.

Proof. Assertion a) of Proposition 4 implies that if equation (10) is true, then

$$
T_{e^{i k \theta} f_{k}} T_{e^{i p \theta} \phi}=T_{e^{i p \theta} \phi} T_{e^{i k \theta} f_{k}} \text {, for all } k \in \mathbb{Z} .
$$

Thus i) is a direct consequence of assertion b) of Proposition 4.

Now, to prove ii), let $k$ be a positive integer such that $\left(T_{e^{i \theta} \psi}\right)^{k}$ is a Toeplitz operator. Then $\left(T_{e^{i \theta} \psi}\right)^{k}$ is a quasihomogeneous Toeplitz operator of degree $k$ which commutes with $T_{e^{i p \theta} \phi}$. So, if $f_{k}$ is not identically equal to zero, then $f_{k}$ is a bounded nonzero radial function such that $T_{e^{i k \theta} f_{k}}$ commutes with $T_{e^{i p \theta} \phi}$. Thus, assertion c) of Proposition 4 implies that $T_{e^{i k \theta} f_{k}} \equiv\left(T_{e^{i \theta} \psi}\right)^{k}$.

Finally, let $k$ be a positive integer such that $\left(T_{e^{i \theta} \psi}\right)^{k}$ is not a Toeplitz operator and suppose that there exists a nonzero bounded radial function $f_{k}$ such that $T_{e^{i k \theta} f_{k}}$ commutes with $T_{e^{i p \theta} \phi}$. Then Proposition 7 implies that

$$
\left(T_{e^{i k \theta} f_{k}}\right)^{p} \equiv\left(T_{e^{i p \theta} \phi}\right)^{k} .
$$

Thus $\left(T_{e^{i k \theta} f_{k}}\right)^{p} \equiv\left(T_{e^{i \theta} \psi}\right)^{k p}$ and Proposition 9 implies that $T_{e^{i k \theta} f_{k}} \equiv\left(T_{e^{i \theta} \psi}\right)^{k}$, which contradicts our hypothesis. This proves iii).

Before starting with corollaries, we state an interesting theorem which follows from [5] and give an idea of its proof. In fact we will apply this theorem to see that if $p$ is any positive integer and $m$ is any nonnegative integer, then the Toeplitz operator $T_{e^{i p \theta} r^{m}}$ always has a T- $p^{t h}$ root. 
Theorem 14. Let $p \geq 1$ and $m \geq 0$ be two integers. For all integers $s$, such that $1 \leq s<p$, there exists a unique bounded radial function $\psi$ such that

$$
T_{e^{i s \theta} \psi} T_{e^{i p \theta} r^{m}}=T_{e^{i p \theta} r^{m}} T_{e^{i s \theta} \psi} .
$$

Proof. (This is a slight variation of the proof found in [5].) If $m \geq 0, p \geq 1$ and $1 \leq s<p$, we define the radial functions $f$ and $g$ by

$$
f(r)=2 p r^{2 s}\left(1-r^{2 p}\right)^{-\frac{s}{p}} \quad \text { and } \quad g(r)=2 p r^{m+p}\left(1-r^{2 p}\right)^{\frac{s}{p}-1} .
$$

Let $\psi$ be the radial function defined by

$$
r^{s} \psi=f *_{M} g .
$$

Cučković and Rao prove, using a long rather technical calculation, that $\psi$ is bounded. Here, we will show that $\psi$ satisfies (11). To do this, we need only verify that for $k \in \mathbb{Z}_{+}$

$$
\frac{2 k+2 p+2}{2 k+m+p+2} \widehat{r^{s} \psi}(2 k+2 p+2)=\frac{2 k+2 s+2}{2 k+m+p+2 s+2} \widehat{r^{s} \psi}(2 k+2) .
$$

By (1), we have $\widehat{r^{s} \psi}(2 k+2)=\widehat{f}(2 k+2) \widehat{g}(2 k+2)$. A simple substitution $t=r^{2 p}$ shows that

$$
\widehat{f}(2 k+2)=B\left(\frac{2 k+2 s+2}{2 p}, 1-\frac{s}{p}\right) \quad \text { and } \quad \widehat{g}(2 k+2)=B\left(\frac{2 k+m+p+2}{2 p}, \frac{s}{p}\right),
$$

where $B$ denotes the beta function. Using the well-known identities $B\left(z_{1}, z_{2}\right)=$ $\frac{\Gamma\left(z_{1}\right) \Gamma\left(z_{2}\right)}{\Gamma\left(z_{1}+z_{2}\right)}$ and $\Gamma(1+z)=z \Gamma(z)$, where $\Gamma$ is the gamma function, it is easy to see that

$$
\widehat{r^{s} \psi}(2 k+2 p+2)=\frac{(2 k+2 s+2)(2 k+m+p+2)}{(2 k+2 p+2)(2 k+m+p+2 s+2)} \widehat{r^{s} \psi}(2 k+2),
$$

which finishes the proof.

Remark 15. i) It is trivial that $T_{e^{i p \theta} r^{m}}$ commutes with itself. So, if $p=s$, assertion c) of Proposition 4 implies that $\psi \equiv r^{m}$.

ii) We wish to highlight the following case. If $m=(2 n+1) p$ for $n \in \mathbb{N}$, then the function $\psi$ exists for all $s \in \mathbb{N}$. In fact, if we substitute $m=(2 n+1) p$ in (12) and use Theorem 1, we obtain for all $z \in \Pi$,

$$
\frac{\widehat{r^{s} \psi}(z+2 p)}{\widehat{r^{s} \psi}(z)}=\frac{F(z+2 p)}{F(z)} \text {, where } F(z)=\frac{\Gamma\left(\frac{z+2 s}{2 p}\right) \Gamma\left(\frac{z}{2 p}+n+1\right)}{\Gamma\left(\frac{z}{2 p}+1\right) \Gamma\left(\frac{z+2 s}{2 p}+n+1\right)} \text {. }
$$

Now, using the identity $\Gamma(1+z)=z \Gamma(z)$ repeatedly, we have

$$
F(z)=2 p \frac{\prod_{j=0}^{n-1}(z+2 j p+2 p)}{\prod_{j=0}^{n}(z+2 j p+2 s)},
$$

which is a proper fraction in $z$ and can be written as

$$
F(z)=\sum_{j=0}^{n} \frac{a_{j}}{z+2 j p+2 s} .
$$

Since $\frac{1}{z+2 j p+2 s}=\widehat{r^{2 j p+2} s}(z)$, it follows by Lemma 6 that

$$
\widehat{r^{s} \psi}(z) \equiv \sum_{j=0}^{n} a_{j} \widehat{r^{2 j p+2} s}(z)
$$


where the $a_{j}$ are defined by (13), and so Theorem 1 implies that

$$
\psi(r) \equiv \sum_{j=0}^{n} a_{j} r^{2 j p+s} .
$$

Next, we give some easy but interesting consequences of Theorem 14 .

Corollary 16. For all integers $m \geq 0, p \geq 1$, and $s \geq 1$ there exists a bounded radial function $\psi$ such that $\left(T_{e^{i s \theta} \psi}\right)^{\bar{p}} \equiv T_{e^{i p s \theta} r^{m}}$.

Proof. Let $m \geq 0, p \geq 1$, and $s \geq 1$ be integers. Theorem 14 implies that there exists a bounded radial function $\psi$ such that

$$
T_{e^{i s \theta} \psi} T_{e^{i p s \theta} r^{m}}=T_{e^{i p s \theta} r^{m}} T_{e^{i s \theta} \psi} .
$$

Using Proposition 7 we have $\left(T_{e^{i s \theta} \psi}\right)^{p s} \equiv\left(T_{e^{i p s \theta} r^{m}}\right)^{s}$ and so, an application of Proposition 9 finishes the proof.

In 4, Brown and Halmos studied multiplicativity of Toeplitz operators on the Hardy space and showed that the product of two Toeplitz operators $T_{f}$ and $T_{g}$ is equal to a third Toeplitz operator $T_{h}$ for some $f, g$ and $h$ in $L^{\infty}(\mathbb{T})$ if and only if $f$ is conjugate analytic or $g$ is analytic, that is, hardly ever. The question of when the product of two Toeplitz operators on the Bergman space is equal to a third is much more complicated and still open. Most work on this question shows that it is not often true that the product of two Toeplitz operators is a Toeplitz operator (see [1] and [6]). But, below, we show that, for certain nontrivial Toeplitz operators $T_{e^{i \theta} \psi}$, not only is $\left(T_{e^{i \theta} \psi}\right)^{2}$ equal to a Toeplitz operator, but there exists a positive integer $k$ such that $\left(T_{e^{i \theta} \psi}\right)^{i}$ is a Toeplitz operator for all positive integers $i \leq k$.

Corollary 17. Let $m \geq 0$ and $p \geq 1$ be two integers. If $T_{e^{i p \theta} r_{m}}$ has a T-p ${ }^{\text {th }}$ root $T_{e^{i \theta} \psi}$ then, for all integers $k$ with $1 \leq k \leq p$, the product $\left(T_{e^{i \theta} \psi}\right)^{k}$ is a Toeplitz operator.

Proof. Let $k$ be an integer such that $1 \leq k \leq p$. By Theorem 14 we know that there exists a bounded radial function $\phi$ such that $T_{e^{i k \theta} \phi}$ commutes with $T_{e^{i p \theta} r^{m}}$. So, Proposition 7 implies that

$$
\left(T_{e^{i k \theta} \phi}\right)^{p} \equiv\left(T_{e^{i p \theta} r^{m}}\right)^{k}
$$

Thus $\left(T_{e^{i k \theta} \phi}\right)^{p}=\left(T_{e^{i \theta} \psi}\right)^{k p}$ since $T_{e^{i \theta} \psi}$ is the T- $p^{t h}$ root of $T_{e^{i p \theta} r^{m}}$, and so Proposition 9 finishes the proof.

It is easily seen that if $f$ is a bounded analytic function on $\mathbb{D}$, then $T_{f}$ is just a multiplication operator. Thus for any integer $k \geq 1$, it is clear that $\left(T_{f}\right)^{k}$ is a Toeplitz operator of symbol $f^{k}$. By taking adjoints, we can see that the powers of a Toeplitz operator with conjugate analytic symbol are also Toeplitz operators. These are the trivial cases. The next corollary says there are nontrivial symbols $f$ such that $\left(T_{f}\right)^{k}$ is always a Toeplitz operator for all integers $k \geq 1$.

Corollary 18. There exist bounded radial functions $\psi$ such that for all integers $k \geq 1$ the product $\left(T_{e^{i \theta} \psi}\right)^{k}$ is still a Toeplitz operator. 
Proof. Let $n \geq 0$ and $p \geq 1$ be two integers. By Theorem 14 we know that the Toeplitz operator $T_{e^{i p \theta} r^{(2 n+1) p}}$ has a T- $p^{t h}$ root $T_{e^{i \theta} \psi}$, where $\psi$ is a bounded radial function. Moreover the assertion ii) of Remark 15 tells us that, for all integers $k \geq 1$, there exists a bounded radial function $\psi_{k}$ such that $T_{e^{i k \theta} \psi_{k}}$ commutes

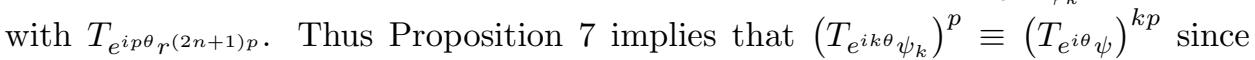
$T_{e^{i p \theta} r^{(2 n+1) p}}=\left(T_{e^{i \theta} \psi}\right)^{p}$ and, again, Proposition 9 finishes the proof.

\section{ACKNOWLEDGMENTS}

I am thankful to Elizabeth Strouse my thesis supervisor for helpful discussions and suggestions concerning the results of this paper.

\section{REFERENCES}

[1] P. Ahern, Z̆. C̆učković, A Theorem of Brown-Halmos Type for Bergman Space Toeplitz Operators, J. Funct. Anal. 187 (2001), 200-210. MR:1867348(2002h:47040)

[2] S. Axler, Z̆. Čučković, Commuting Toeplitz operators with harmonic symbols, Integral Equations and Operator Theory 14 (1991), 1-12. MR1079815 (92f:47018)

[3] S. Axler, Z̆. Cučković, N. V. Rao, Commutants of analytic Toeplitz operators on the Bergman space, Proc. Amer. Math. Soc. 128 (2000), 1951-1953. MR1694299 (2000m:47035)

[4] A. Brown, P. Halmos, Algebraic properties of Toeplitz operators, J. Reine Angew. Math. 213 (1964), 89-102. MR0160136 (28:3350)

[5] Z̆. Cučcković, N. V. Rao, Mellin transform, monomial symbols, and commuting Toeplitz operators, J. Funct. Anal. 154 (1) (1998), 195-214. MR1616532 (99f:47033)

[6] I. Louhichi, E. Strouse, L. Zakariasy, Products of Toeplitz operators on the Bergman space, Integral Equations and Operator Theory. Published on line: 1 October 2005.

[7] I. Louhichi, L. Zakariasy, On Toeplitz operators with quasihomogeneous symbols, Archiv der Mathematik. Volume 85, Number 3 (2005), 248-257. MR2172383 (2006e:47061)

[8] R. Remmert, Classical Topics in Complex Function Theory, Graduate Texts in Mathematics, Springer, New York, 1998. MR1483074 (98g:30002)

UFR Mathématiques et Informatique, Université Bordeaux 1, 351 COURS de la Libération, 33405 Talence, France

E-mail address: louhichi@math.u-bordeaux1.fr 